M. EL KHATIB, R. A. M. SERAFIM, G. A. MOLANDER* (UNIVERSITY OF PENNSYLVANIA, USA AND UNIVERSITY OF SÃO PAULO, BRAZIL)

$\alpha$-Arylation/Heteroarylation of Chiral $\alpha$-Aminomethyltrifluoroborates by Synergistic Iridium Photoredox/Nickel

Cross-Coupling Catalysis

Angew. Chem. Int. Ed. 2016, 55, 254-258.

\section{Photoredox-Generated $\alpha$-Amino Radicals Used in (Hetero)Arylation}

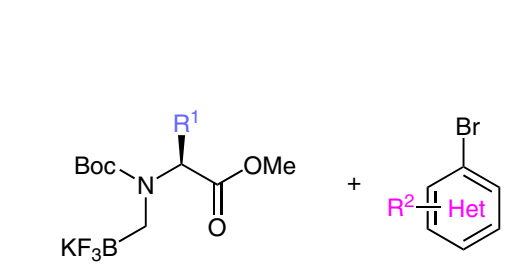

$\operatorname{Ir}\left[\mathrm{dFCF}_{3} \mathrm{ppy}_{2}(\mathrm{bpy}) \mathrm{PF}_{6}(2 \mathrm{~mol} \%)\right.$ $\mathrm{Ni}\left(\mathrm{NO}_{3}\right)_{2} \cdot \mathrm{H}_{2} \mathrm{O}(5 \mathrm{~mol} \%)$

Selected examples:<smiles>COC(=O)CN(Cc1ccc(NC(C)=O)cc1)C(=O)OCc1ccccc1</smiles><smiles>COC(=O)C(C)N(Cc1ccc(S(N)(=O)=O)s1)C(=O)c1ccccc1</smiles>

$78 \%$ yield<smiles>CCC(C)C(C(=O)OC)N(Cc1ccc(-c2nnco2)cc1)C(=O)c1ccccc1</smiles>

$80 \%$ yield
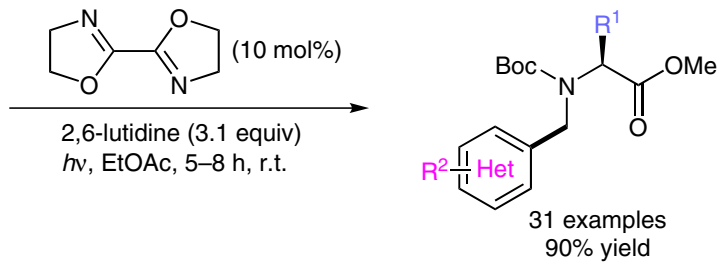<smiles>COC(=O)CN(Cc1ccc(C(=O)OC)cc1)C(=O)c1ccccc1</smiles><smiles>COC(=O)C(C)N(Cc1cccc(O)c1)C(=O)c1ccccc1</smiles>

$78 \%$ yield<smiles>COC(=O)C(Cc1ccc(OC)cc1)N(Cc1cncnc1)C(=O)c1ccccc1</smiles>

Category

Metal-Mediated

Synthesis

\section{Key words}

iridium

nickel

photochemistry

amino acids

Synfact of the ond
Significance: Molander and co-workers report the arylation of chiral $\alpha$-aminomethyltrifluoroborates by a synergistic dual catalysis strategy using iridium photoredox and nickel cross-coupling. $\alpha$-Amino radical generation from the chiral $\mathrm{N}$-trifluoroboratomethyl salt, followed by arylation, leads to enantiopure benzylic amines in high yields.
Comment: This new Csp ${ }^{3}-\mathrm{Csp}^{3}$ bond-formation process uses abundant and inexpensive amino acids, and also has the advantage of the stability and storability of its reagents. Moreover, the reactions proceed at room temperature, under mild conditions.

SYNFACTS Contributors: Paul Knochel, Sarah Fernandez 